\title{
SEGURIDAD ALIMENTARIA Y SU RELACIÓN CON FACTORES SOCIECONÓMICOS: CASO FAMILIA DE PRODUCTORES DE QUINUA DE LA PARROQUIA SAN ISIDRO, CANTÓN ESPEJO, PROVINCIA DEL CARCHI
}

\author{
Priscila Maldonado P.
}

\section{RESUMEN}

En la actualidad muchas comunidades rurales de Ecuador sufren de escasez de alimentos que componen la canasta familiar básica. De tal manera que la Organización de Naciones Unidas para la Agricultura y Alimentación (FAO) conceptualiza a la seguridad alimentaria como la suficiencia permanente de alimentos a los que tiene derecho la población para llevar una vida sana y activa. Si bien este concepto se lo conoce desde 1996 por falta de estrategias claras y precisas no se lo ha podido desarrollar.

Esta investigación analizó la relación que existe entre los factores socieconómicos y la seguridad alimentaria de 78 familias que se dedican a la produc- ción de quinua como principal actividad económica, tomando como premisa el estudio realizado en Risalda Colombia donde la línea base nutricional en menores de 5 años evidenció que los niños del campo no se beneficiaban por ser hijos de familias productoras de alimentos; por el contrario en algunos municipios varios niños presentaban las cifras más altas de desnutrición crónica en un porcentaje de 28,6\% (Martínez J. , 2009).

Los resultados nos permiten establecer que no todos los indicadores socioeconómicos se relacionan con la seguridad alimentaria medida con el instrumento ELCSA.

Palabras clave: quinua, disponibilidad, autoconsumo, indicadores

${ }^{1}$ Pontificia Universidad Católica del Ecuador, Facultad de Enfermería, Quito, Ecuador (bpmaldonado@puce.edu.ec). 


\begin{abstract}
Today many rural communities in Ecuador suffer from food shortage that makes up the basic family basket. Therefore, the United Nations Food and Agriculture Organization (FAO) conceptualizes food security as permanent sufficiency of food, which should be the right of people to be able to live a healthy and active life; although this concept has been known since 1996 due to lack of clear and precise strategies it has not been able to be developed.
\end{abstract}

This research analyzed the relation between socioeconomic factors and food security of 78 families dedicated to the production of quinoa as the main economic activity, taking as its premise the study in Risalda Colombia where the nutritional baseline in children under 5 years showed that rural children did not receive any benefit for being children of farming families. However some children in some municipalities had the highest rates of chronic malnutrition in a percentage of $28.6 \%$ (J. Martinez, 2009).

The results allow us to establish that not all economic indicators are related to food security meassured with the ELCSA instrument.

Keys words: Quinoa, availability, consumption, indicators

\section{INTRODUCCIÓN}

En América Latina y el Caribe la Seguridad Alimentaria ha tenido un notable impulso en los últimos diez años; varios elementos han favorecido el proceso de cambio en torno a una institucionalidad que promueve la protección de los derechos económicos, sociales y culturales, especialmente el derecho a la alimentación adecuada y suficiente.

El compromiso del Ecuador por garantizar seguridad alimentaria en la población nace en la Cumbre Mundial de la Alimentación celebrada en el año 1996, en la cual Ecuador se comprome- tió a desarrollar procesos que permitan garantizar un entorno político, social y económico propicio para reducir el número de personas con hambre en el país; aplicar políticas que tengan como objetivo erradicar la pobreza; adoptar políticas y prácticas participativas para el desarrollo alimentario; y construir planes de acción con todos los niveles de gobierno en cooperación para combatir el hambre. Estos acuerdos constan en el Decreto Ejecutivo n. ${ }^{\circ} 1039$ el 9 de enero de 1998, que declara a la Seguridad Alimentaria de la población ecuatoriana como Política de Estado. 


\section{SOBERANÍA ALIMENTARIA}

Actualmente el término Soberanía Alimentaria está en construcción, este dependerá de la situación de cada país o localidad; en sus inicios en 1996 fue impulsado por La Vía Campesina, en el contexto de la Cúpula Mundial sobre la Alimentación (CMA) realizada en Roma por la FAO. En este evento el debate oficial giraba en torno de la noción de la seguridad alimentaria, reafirmándola como "El derecho de toda persona a tener acceso a alimentos sanos y nutritivos, en consonancia con el derecho a una alimentación apropiada y con el derecho fundamental de no pasar hambre". Sin embargo, la Organización Mundial del Comercio (OMC) enfocó este principio a la liberalización del comercio de alimentos, motivo que generó controversia con las organizaciones campesinas.

En el Plan Nacional del Buen Vivir 2013-2017 se enuncia acerca de la Soberanía Alimentaria que esta se sustenta en el reconocimiento del derecho a la alimentación sana, nutritiva y culturalmente apropiada, para lo cual es importante incidir tanto en las condiciones de producción y distribución como en el consumo de alimentos. La Soberanía Alimentaria implica recuperar el rol de la sociedad para decidir: qué producir, cómo producir, dónde producir y para quién producir (SENPLADES, 2009).

\section{ESCALA LATINOAMERICANA Y CARIBEÑA DE SEGURIDAD ALIMENTARIA (ELCSA)}

La Escala Latinoamericana y Caribeña de Seguridad Alimentaria (ELCSA) pertenece a la categoría de las escalas de medición de inseguridad alimentaria que se basan en la experiencia en los hogares con esta condición (Tapajós, Quiroga, Pereira, \& Pinto, 2010). La ELCSA es un instrumento que ha demostrado ser muy eficaz y además cuenta con un alto grado de confiabilidad. Esto se debe al enfoque inclusivo y multisectorial que se siguió para el desarrollo, validación, y diseminación del uso de la escala, su metodología es simple, ya que cuenta con quince preguntas de carácter dicotómico, donde sus respuestas son: "sí" o "no". Estas preguntas están dirigidas al jefe/a de familia o a la persona encargada de preparar los alimentos en hogares conformados por adultos e integrantes menores de 18 años. Las primeras ocho preguntas están destinadas a medir solamente el grado de inseguridad alimentaria en hogares que no se encuentren conformados por menores de 18 años; en caso de tener integrantes menores de 18 años; en el hogar se deben responder las 15 preguntas (SESAN 
\& FAO, 2011). El período de referencia para las preguntas son los tres meses previos a la aplicación de la encuesta (Melgar, 2011).

\section{Tabla 1. Categorías de Inseguridad Alimentaria según el número de respuestas positivas en la encuesta ELCSA}

\begin{tabular}{|lcc|}
\hline CATEGORÍA & $\begin{array}{c}\text { NÚMERO DE RESPUESTAS POSITIVAS } \\
\text { Hogares con menores } \\
\text { de } 18 \text { años }\end{array}$ & $\begin{array}{c}\text { Hogares sin menores } \\
\text { de18 años }\end{array}$ \\
\hline Seguridad Alimentaria & 0 & 0 \\
Inseguridad Leve & $1-5 / 15$ & $1-3 / 8$ \\
Inseguridad Moderada & $6-10 / 15$ & $4-6 / 8$ \\
Inseguridad Severa & $11-15 / 15$ & $7-8 / 8$ \\
\hline
\end{tabular}

Fuente: SESAN \& FAO, 2011

Elaborado por SESAN \& FAO, 2011

\section{MATERIALES Y MÉTODOS}

Esta investigación se basa en un estudio observacional, mediante estadística descriptiva. El corte es de tipo transversal, como fuentes primarias se aplicaron encuestas de condiciones de vida, dentro de las cuales se indagó: el acceso a electricidad, al agua potable, al alcantarillado, discapacidad, analfabetismo, principal rama de actividad, nivel de instrucción, número de horas trabajadas, tipo de vivienda, número de personas por vivienda, vía de acceso principal a la vivienda, material del piso, ingreso familiar mensual promedio, procedencia del agua, destino de la producción, suficiencia del consumo de alimentos. Para establecer el grado de Seguridad Alimentaria se aplicó la Encuesta de la Escala Latinoamericana y Caribeña de
Seguridad Alimentaria (ELCSA), mediante criterios como: seguridad, inseguridad leve, inseguridad moderada e inseguridad severa.

Para la selección de la muestra se consideró a los productores de quinua con una producción máxima de 10 quintales anuales del producto y que tengan niños menores de 5 años como miembros de familia. Estos criterios totalizaron una muestra de 78 productores dividido en las comunidades de Carlisama ( $\mathrm{N}=23)$, Puchues ( $N=14)$ San Isidro ( $N=21)$ Chitacaspi $(\mathrm{N}=20)$.

El análisis de datos se realizó mediante la prueba estadística no paramétrica Chi Cuadrado de Pearson aplicando 
el programa estadístico IBM SPSS Statis- Machines Corp. IBM, 2012). tics versión 21.0 (International Business

\section{RESULTADOS}

Se analizaron varios indicadores establecer que 8 indicadores tienen resocieconómicos relacionados con el lación. nivel de seguridad alimentaria y se pudo

\section{Tabla 2. Resultados de la relación de indicadores socioeconómicos y el grado de seguridad alimentaria}

\begin{tabular}{|lr|}
\hline $\begin{array}{l}\text { Indicadores asociados relacionas } \\
\text { con el grado de Seguridad Alimentaria }\end{array}$ & Correlación \\
\hline Acceso a electricidad & $\left(X^{2}=1,88 ; p=0,59\right)$ \\
Acceso al agua potable & $\left(X^{2}=4,25 ; p=0,02\right) *$ \\
Acceso al alcantarillado & $\left(X^{2}=3,58 ; p=0,031\right)$ \\
Discapacidad & $\left(X^{2}=1,88 ; p=0,59\right)$ \\
Analfabetismo & $\left(X^{2}=3,71 ; p=0,29\right)$ \\
Principal rama de actividad & $\left(X^{2}=22,85 ; p=0,52\right)$ \\
Nivel de instrucción & $\left(X^{2}=16,14 ; p=0,37\right)$ \\
Número de horas trabajadas & $\left(X^{2}=16,38 ; p=0,01\right) *$ \\
Tipo de vivienda & $\left(X^{2}=16,53 ; p=0,01\right) *$ \\
Número de personas por vivienda & $\left(X^{2}=12,24 ; p=0,02\right) *$ \\
Vías de Acceso principal a la vivienda & $\left(X^{2}=11,27 ; p=0,04\right)$ \\
Material del piso & $\left(X^{2}=7,96 ; p=0,92\right)$ \\
Ingreso familiar mensual promedio & $\left(X^{2}=11,21 ; p=0,02\right) *$ \\
Procedencia del agua & $\left(X^{2}=7,19 ; p=0,61\right)$ \\
Destino de la producción & $\left(X^{2}=8,260 ; p=0,02\right) *$ \\
Suficiencia del consumo de alimentos & $\left(X^{2}=92,24 ; p=0,00\right) *$ \\
\hline Señala asociación relación entre el indicador socioeconómico \\
y el grado de seguridad alimentaria &
\end{tabular}

Al calcular la relación entre el acceso a servicios básicos y el grado de seguridad alimentaria se pudo determinar lo siguiente: El acceso a electricidad no tiene relación con el grado de seguridad alimentaria ( $\left.X^{2}=1,881 ; p=0,598\right)$, en cuanto al acceso de agua potable se establece que existe relación estadística $\left(X^{2}=4,255 ; p=0,023\right)$; respecto al acceso al alcantarillado existe relación estadística $\left(X^{2}=3,584 ; p=0,031\right)$. Figura 1 . 
En los hogares cuyos miembros tienen más acceso al mercado laboral presentan menor probabilidad de inaccesibilidad a una cantidad suficiente de alimentos, esto dependerá de cómo esté reconocido económicamente ese trabajo. Según Álvarez si un adulto en el hogar tiene trabajo remunerado, la probabilidad que el hogar sufra de inseguridad alimentaria se reduce en un 2,8\% a nivel rural.

En un estudio realizado por Rojas (2014)se establece que los hogares en los cuales los miembros de familia ganan menos de 10 dólares al día, sobrellevan un alto grado de inseguridad alimentaria severa; esta situación disminuye cuando las familias perciben mayores ingresos. Figura 2.

Respecto a la tenencia de vivienda, en el estudio de caso realizado por Rose (1998) en Estados Unidos, se determinó que los hogares que poseen vivienda propia, tienen menor probabilidad de sufrir inseguridad alimentaria, en cambio Calero en un estudio sobre Seguridad Alimentaria y Nutricional en Ecuador, establece que según la ECV 2005-2006 apenas el 40,6\% de los hogares que no acceden a una cantidad suficiente de alimentos poseen vivienda propia, uno de los factores a los cuales se atribuye este fenómeno es a la utilización del ingreso familiar en el pago por la vivienda, colocando como segunda opción la alimentación familiar.
En el 2004 se reportaron varios estudios realizados en diversos países que aportan información acerca de la relación entre tener derechos de propiedad de la tierra con el acceso a créditos y a montos de créditos superiores cuando existe la garantía de la tierra; además se estableció la correlación entre la desigualdad de la propiedad de la tierra y el grado de pobreza en regiones como el Sur de Asia, Sur de África y América Latina. Figura 3.

Respecto al número de personas que componen el hogar y considerando que la seguridad alimentaria se fundamenta en garantizar el acceso a los alimentos a todos los miembros del hogar, mientras mayor es la cantidad de miembros de la familia más recursos alimentarios se necesitan para satisfacer las necesidades energéticas.

Según Calero los hogares que presentan mayores indices de inseguridad alimentaria son más numerosos, tienen mas niños menores de cinco años y una gran proporción de miembros dependientes. Existe asociación estadísticamente significativa entre el número de integrantes de un hogar y el grado de seguridad alimentaria $\left(X^{2}=12,244 ; p\right.$ $=0,02)$. Figura 4 .

En un estudio realizado sobre seguridad alimentaria en el departamento de Antioquia en Colombia, se encontró 
que el promedio de integrantes de los hogares fue similar en la zona urbana $(4,6 \pm 2,1)$ y rural $(4,7 \pm 2,1)(p>0,05)$. En este estudio tanto en el área urbana como rural, los hogares tienden a ser más pequeños, incluso en población de pequeños productores de alimentos para el autoconsumo. Esto implica que la población rural asume algunas conductas similares a la urbana, como por ejemplo, la planificación familiar y la determinación de tener menos hijos. El hecho de que los hogares sean pequeños, la producción de alimentos para el autoconsumo mejorará las condiciones de seguridad alimentaria, ya que se representa en mayor disponibilidad per cápita de alimentos por miembro de familia.

El autoconsumo de alimentos cultivados representa una ventaja para la población, pues contribuye a que exista seguridad alimentaria, ya que las familias tienen mayor disponibilidad y accesibilidad de alimentos, y no implica un costo extra, lo que se traslada en beneficios a la economía familiar.

En un estudio realizado por la FAO (2008) respecto al incremento de precios de los alimentos y la respuesta de los hogares, se estableció que de nueve paises en desarrollo cerca del $75 \%$ de hogares rurales son compradores netos ${ }^{2}$ de alimentos.
El Banco Mundial (2007) realizó un estudio sobre la "Insuficiencia Nutricional en Ecuador", en los resultados obtenidos se determinó que los niños pertenecientes a familias de pequeños agrícultores a nivel rural presentan un estado nutricional deficiente; según el estudio esto se atribuye al aislamiento de estas zonas y al incremento en la cadena de intermediarios, que provocan encarecer a los alimentos.

Se puede establecer que existe asociación entre el destino de la producción de alimentos con el grado de seguridad alimentaria $\left(X^{2}=8,260 ; p=0,022\right)$.

Según los resultados obtenidos, 44 de 78 familias que venden toda la producción de cultivos, padecen de inseguridad alimentaria, los datos emitidos por Alvear en su estudio Seguridad y Soberanía en Ecuador denotan que el cambio de patrones alimentarios contribuye a la revalorización de cultivos tradicionales altamente nutritivos, ocasionando deficiencias alimentarias como la desnutrición y subnutrición. Figura 5.

El autoconsumo tiene una repercusión importante en la seguridad alimentaria nutricional en los hogares; en un estudio realizado en la ciudad de Fómeque, sobre autoconsumo y seguridad alimentaria, se constató que el autocon-

\footnotetext{
${ }^{2}$ Comprador neto. Se considera comprador neto cuando el valor de los productos básicos producidos en el hogar es menor que la cantidad que consume.
} 
sumo tenía una participación que osciló entre el $21-82 \%$ del costo de la canasta básica y representó el 43\% del ingreso familiar del agricultor .

Otro indicador importante es la suficiencia alimentaria; esta se calcula a través de la kilocalorías totales consumidas en el hogar dividido para las kilocalorías requeridas según la hoja de balance de alimentos de cada país; el valor resultante se lo transforma a porcentaje y se determina si es suficiente, insuficiente o crítica. Entre los factores que afectan a la suficiencia alimentaria están el empleo, los ingresos promedio, la pobreza y la desigualdad.

En los resultados se puede observar que 55 de las 78 familias encuestadas presentan insuficiencia alimentaria; al relacionar este indicador con el grado de seguridad alimentaria se establece una asociación estadística $\left(X^{2}=92,24\right.$; $p=0,00)$. La suficiencia alimentaria es un dato real reportado por la población al estar vinculada con el grado de seguridad alimentaria medida por la ELCSA se puede obtener un panorama real de la percepción con la situación.

La suficiencia alimentaria es un indicador de extrema relevancia para la seguridad alimentaria, ya que es el indicador más directo para relacionar la deficencia calórica consumida con la requerida por cada miembro de las familias de productores. Figura 6.

Para visualizar en qué lugar de inseguridad alimentaria se encuentran los productores de quinua se realizó una comparación a nivel nacional e internacional.

La Figura 7 muestra claramente que los países considerados desarrollados presentan bajos niveles de inseguridad alimentaria. A medida que se acerca a lo local se incrementa el riesgo de inseguridad alimentaria. Canadá tan solo presenta un $12 \%$ de inseguridad alimentaria, comparado con Antioquia en Colombia que presenta el $80 \%$ de inseguridad; en la parroquia de Sigchos el porcentaje es del 91 \% y por último de las familias productoras de quinua con un $73 \%$, si bien el universo de estudio no es el mismo, se pueden trasponer los resultados para una interpretación, ya que se utiliza el mismo instrumento de medición.

La comparación más real se realiza al tomar los datos de Antioquia y de la familia de productores de quinua de San Isidro, ya que los dos universos dedican su actividad principal a la producción de alimentos, ahí se puede evidenciar la similitud de los datos. 


\section{CONCLUSIONES}

A partir del análisis de los indicadores socieconómicos de las familias de productores de quinua de San Isidro, se pudo establecer que cuentan con servicios básicos y con un nivel de instrucción medio, indicadores relevantes para medir la calidad de vida de una población.

Respecto al ingreso se concluye que tan solo el $15 \%$ de 78 familias encuestadas; acceden a ingresos que permiten cubrir los costos de una canasta básica, en cuanto a las horas destinadas para trabajo el $73 \%$ de los jefes de hogar, trabajan 40 o más horas; esto revela que las horas trabajadas no son bien remuneradas acorde con la resolución del Ministerio del Trabajo que considera el salario mínimo vital en USD 340.

Las familias encuestadas están ubicadas en la zona rural, esta condición se relaciona con el bajo porcentaje de hogares que disponen de vías de acceso en buenas condiciones, este dato limita la disponibilidad de recursos alimentarios en la población.

Indicadores relacionados con las condiciones del hogar como material del piso, cocina exclusiva y material de construcción, denotan el grado de pobreza que tiene una población; en la presente investigación estas no fueron relevantes, ya que los hogares disponían de buenos recursos.

Uno de los indicadores relevantes en este estudio es el destino de la producción de alimentos, el 72,2 \% se reservan para la venta y tan solo un $24,1 \%$ entre venta y consumo, analizando estos valores con la suficiencia alimentaria se evidencia el cambio de patrones alimentarios, las familias destinan los recursos que adquieren de la venta de sus productos a la compra de alimentos que no aportan significativamente a su dieta.

Según la investigación realizada, la inseguridad alimentaria en los hogares de productores de quinua presenta una asociación con el acceso al agua potable, tipo de vivienda, total de horas destinadas al trabajo, vías de acceso, ingreso familiar promedio y suficiencia en el consumo de alimentos.

La Seguridad Alimentaria y Nutricional (SAN) determina en gran medida la calidad de vida de la población de un país y aglutina diversos aspectos de las políticas de Estado, como la educación, la salud, la cultura, el ambiente, la alimentación y nutrición y el acceso a saneamiento básico, entre otros. 


\section{Anexos}

Figura 1. Relación de seguridad alimentaria con los servicios básicos

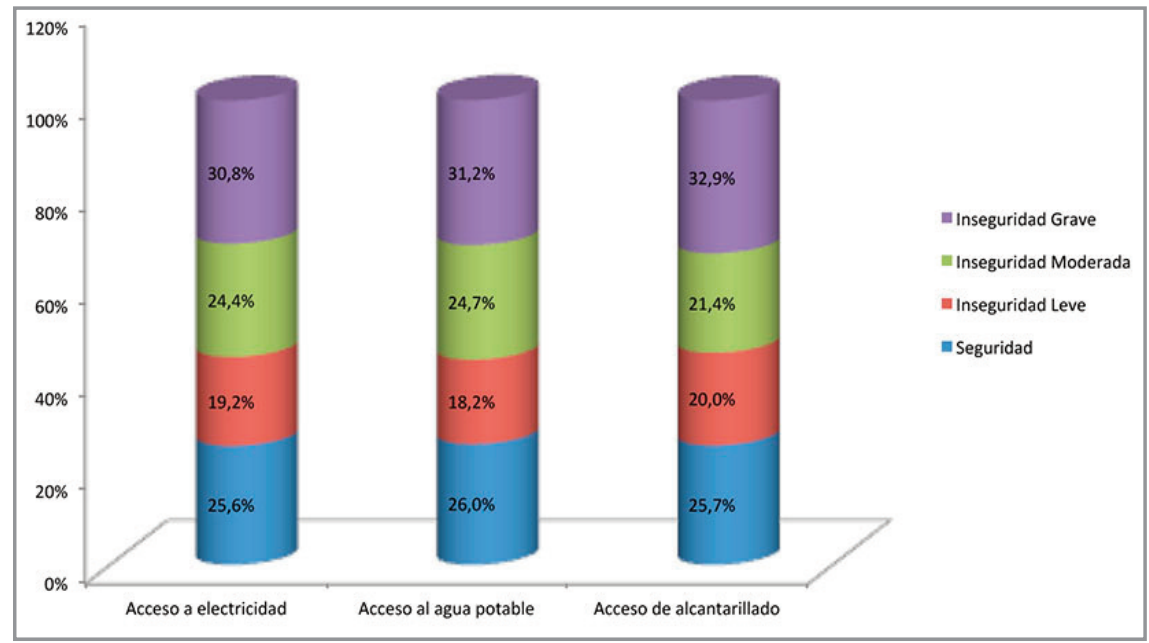

Fuente: Investigación Directa, Encuesta Elaborado por Priscila Maldonado

Figura 2. Relación de seguridad alimentaria con las horas trabajadas por el jefe de hogar

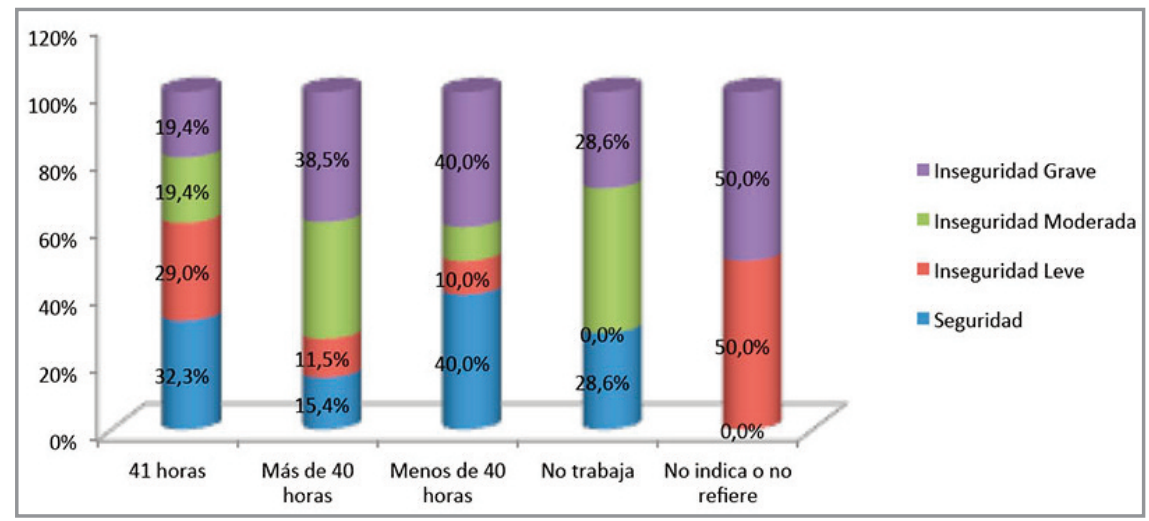

Fuente: Investigación Directa, Encuesta Elaborado por Priscila Maldonado 
Figura 3. Relación de seguridad alimentaria con la tenencia de la vivienda

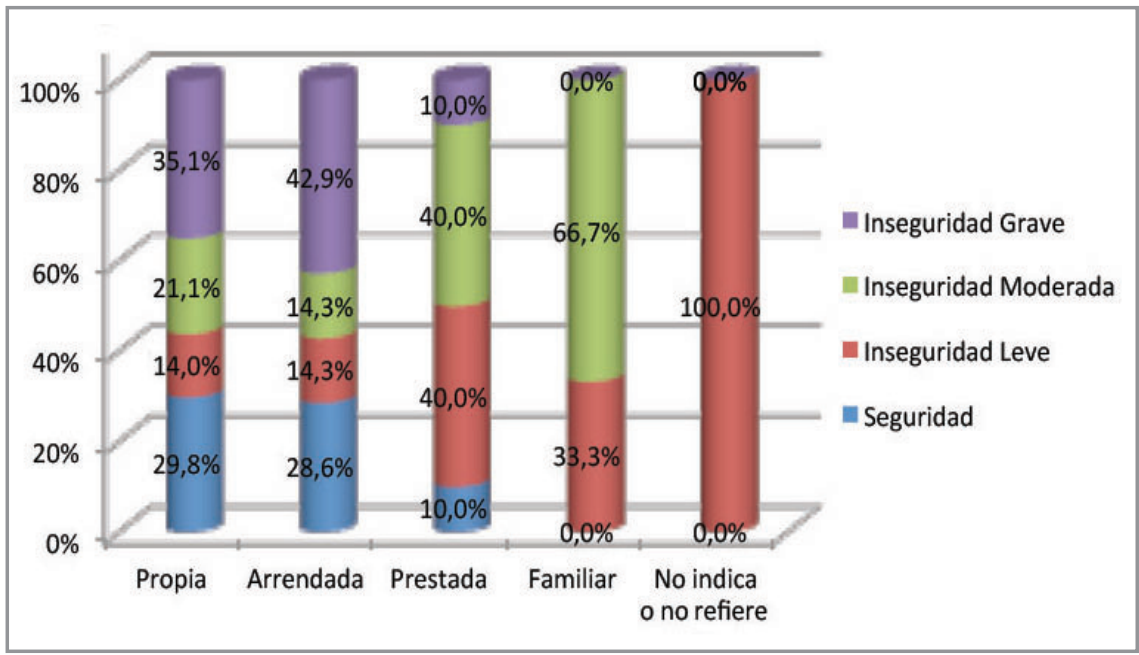

Fuente: Investigación Directa, Encuesta Elaborado por Priscila Maldonado

Figura 4. Relación de seguridad alimentaria con el número de personas por vivienda

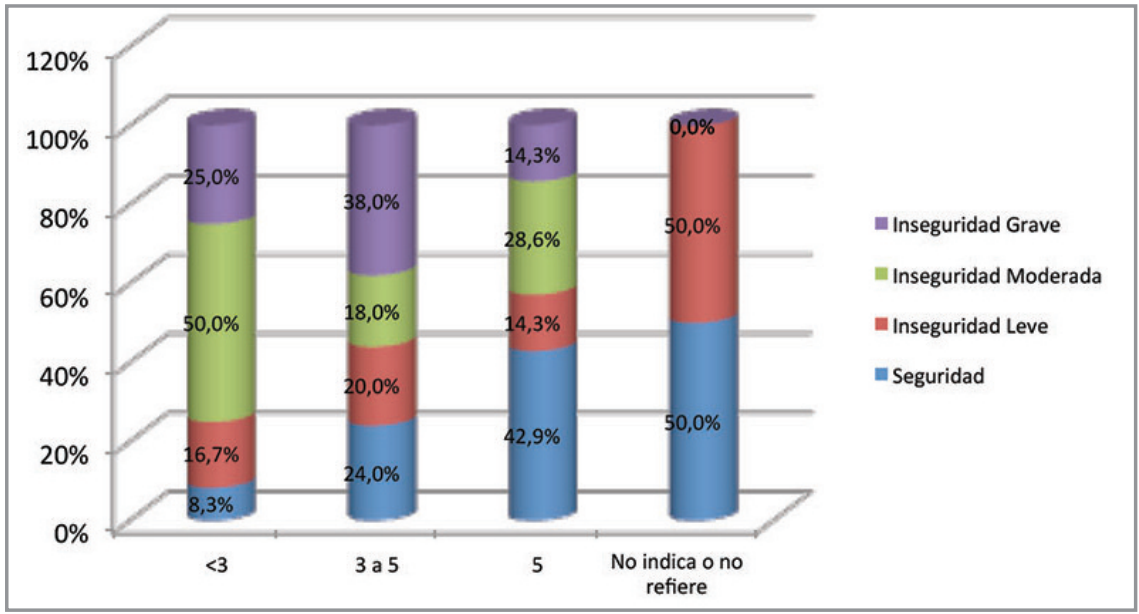

Fuente: Investigación Directa, Encuesta Elaborado por Priscila Maldonado 
Figura 5. Relación de seguridad alimentaria con el destino de la producción de alimentos

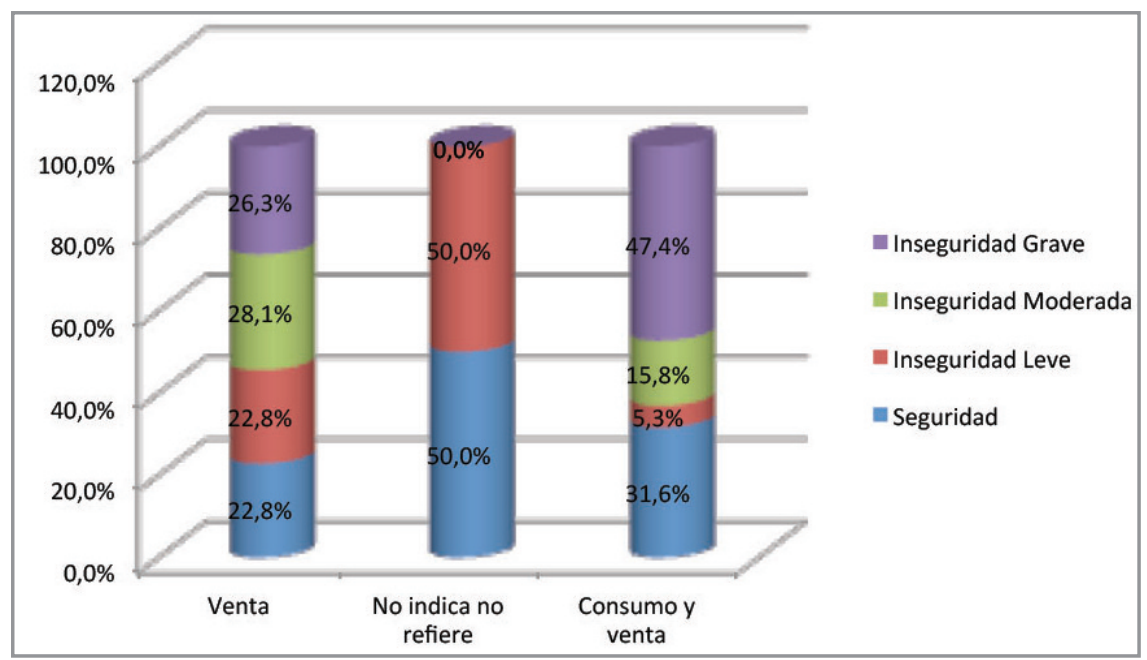

Fuente: Investigación Directa, Encuesta Elaborado por Priscila Maldonado

Figura 6. Relación de seguridad alimentaria con la suficiencia alimentaria

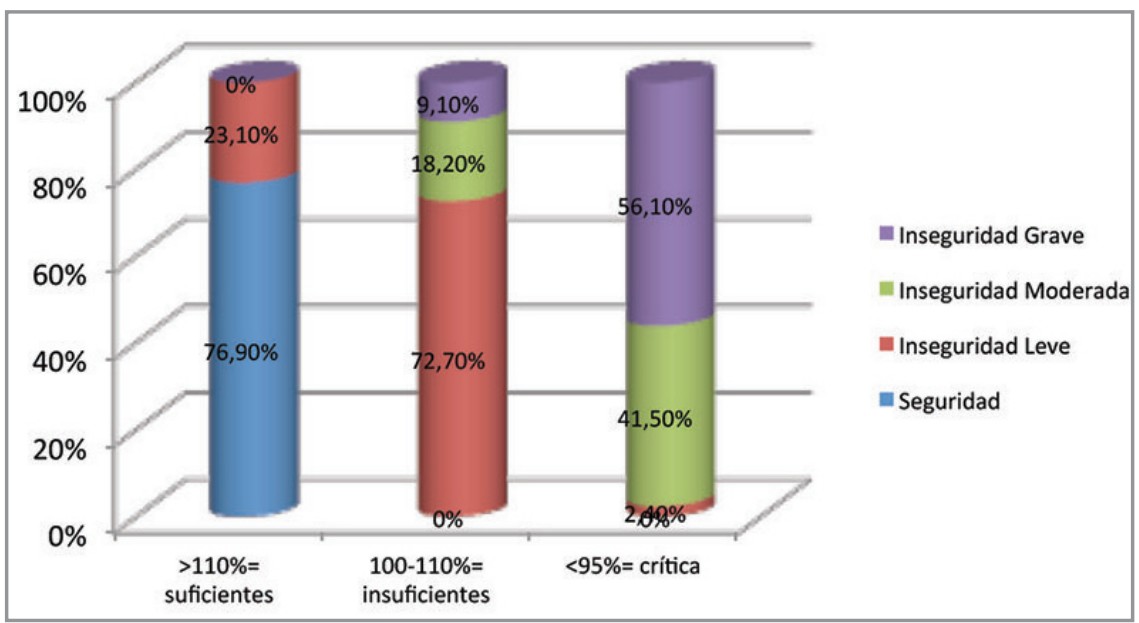

Fuente: Investigación Directa, Encuesta Elaborado por Priscila Maldonado 
ReVista PUCE, ISSN 1012-389X. NúM, 102

Figura 7. Comparación del nivel de seguridad alimentaria en varias localidades

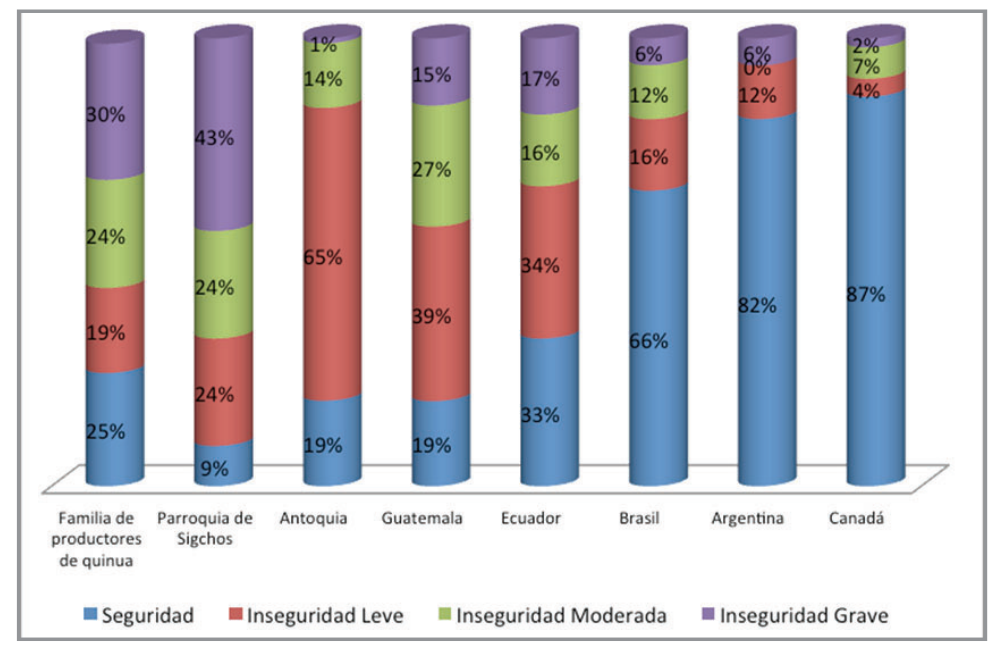

Fuente: Burns, (2004), (Tarasuk, 2012), (FAO, 2002), (Salvia, 2010), (Rojas, 2014) Elaborado por Priscila Maldonado 


\section{BIBLIOGRAFÍA}

Álvarez, M., Mancilla, L., \& Cortéz, J. (2007). Caracterización Socioeconómica y de Seguridad Alimentaria de Los Hogares Productores De Alimentos Para El Autoconsumo, Antioquia-Colombia. Mérida: AGROALIMENTARIA.

Alvear, J. (2012). La Seguridad y Soberanía Alimentaria en el Ecuador. Quito: Aries.

Calero, C. (Octubre de 2011). La Seguridad Alimentaria en Ecuador desde un enfoque de accesibilidad alimentaria. Quito, Pichincha, Ecuador: Abya-Yala.

FAO. (2004). Políticas de desarrollo agrícolas: conceptos y principios. Departamento de Cooperación Técnica. Roma: FAO.

Melgar, H. (2011). Prevalencia de la inseguridad alimentaria en el hogar de Guatemala. Guatemala: FAO.
Menchú, M., \& Santizo, M. (2002). Propuesta de indicadores para la vigilancia de la seguridad alimentaria y nutricional. Guatemala: INCAP/OPS/OMS.

OBSAN-R. (2008). Observatorio Regional de Seguridad Alimentaria y Nutricional, Información de Coyuntura sobre el Alza del Precio de. PRESANCA.

Samaniego, J. (2010). La institucionalidad de la Soberanía y Seguridad Alimentaria en el Ecuador. En M. d. Social, Seguridad Alimentaria y Nutricional del Ecuador (págs. 231 - 241). Ecuador: Aries. 